\title{
Anti-Mullerian Hormone and Follicle Counts as Predictors of Superovulatory Response and Embryo Production in Beef Cattle
}

\author{
Keith Center ${ }^{1}$, Dave Dixon ${ }^{2}$, Charles Looney ${ }^{3}$, Rick Rorie ${ }^{1}$ \\ ${ }^{1}$ Department of Animal Science, University of Arkansas, Fayetteville, AR, USA \\ ${ }^{2}$ Food Animal Veterinary Services, Rensselaer, IN, USA \\ ${ }^{3}$ Ovagenix, Bryan, TX, USA \\ Email: rrorie@uark.edu
}

How to cite this paper: Center, K., Dixon, D., Looney, C. and Rorie, R. (2018) Anti-Mullerian Hormone and Follicle Counts as Predictors of Superovulatory Response and Embryo Production in Beef Cattle. Advances in Reproductive Sciences, 6, 22-33. http://dx.doi.org/10.4236/arsci.2018.61003

Received: December 15, 2017

Accepted: February 24, 2018

Published: February 27, 2018

Copyright $\odot 2018$ by authors and Scientific Research Publishing Inc. This work is licensed under the Creative Commons Attribution International License (CC BY 4.0).

http://creativecommons.org/licenses/by/4.0/

\begin{abstract}
This study evaluated Anti-Mullerian hormone (AMH) and/or follicle counts as predictors of subsequent superovulatory response and embryo production in 79 beef cows. Before superovulation, 3 to $5 \mathrm{~mm}$ follicles presented on the ovaries of donor cows were counted, and blood was collected for measure of serum AMH. Across cows, serum AMH ranged from 0.013 to $0.898 \mathrm{ng} / \mathrm{mL}$, with a mean of $0.293 \mathrm{ng} / \mathrm{mL}$. The distribution of both AMH concentrations and follicle counts were divided into quartiles (AMH Q1 through Q4, with Q1 the lowest and Q4 the highest) for analysis. Donor cows in AMH Q4 had a greater $(\mathrm{P}<0.001)$ number of 3 to $5 \mathrm{~mm}$ follicles at the start of superovulation than did donors in either Q1 or Q2. At embryo collection, cows in AMH Q3 and 4 had more $(\mathrm{P}<0.001)$ palpable corpora lutea $(\mathrm{CL})$ than cows in $\mathrm{AMH}$ $\mathrm{Q} 1$. The mean number of embryos recovered from donor cows in AMH Q4 was greater $(\mathrm{P}<0.001)$ than those recovered from cows in either AMH Q1 or 2, but similar to that of AMH Q3. Analysis indicated that $\mathrm{AMH}$ was positively correlated $(\mathrm{P}<0.001)$ with mean follicles $(\mathrm{r}=0.458), \mathrm{CL}(\mathrm{r}=0.452)$ and embryos recovered $(r=0.430)$. Donor cows with higher follicle counts (F Q3 and $4)$ at the start of superovulation had more $(\mathrm{P}<0.001)$ palpable $\mathrm{CL}$ at embryo collection than donor cows in F Q1 or 2. More $(\mathrm{P}<0.001)$ embryos were recovered from cows with the highest follicle counts (F Q4) as compared with cows having lower (F Q1 and 2) follicle counts. Results confirm that relative AMH concentration was positively correlated with number of small antral follicles in the ovaries of cows; both AMH measure and antral follicle counts might be used to predict subsequent superovulatory response in beef cows.
\end{abstract}




\section{Keywords}

Anti-Mullerian Hormone, Superovulation, Embryos, Cattle

\section{Introduction}

Since the development of cattle superovulation and non-surgical embryo recovery in the 1970s, the unpredictability and variability of the superovulatory response has remained a major obstacle. Hasler [1] reviewed developments that have occurred the past 40 years in the cattle embryo transfer industry, and reported that the average number of transferrable embryos has remained essentially unchanged at about 6 transferable embryos per superovulation and embryo collection. While some females consistently produce a number of embryos, others of similar age, breed, and management perform poorly. The goal of superovulation is to stimulate a number of small antral follicles to grow and mature, resulting in multiple ovulations. Therefore, the pool of small antral follicles available for stimulation should dictate the superovulatory response.

There is an inherent variability in the number of follicles present in the ovaries of heifers. Erickson [2] reported the pool of primordial follicles within the ovaries harvested from heifers under 1 year of age ranged from $\sim 60,000$ to 240,000 . In comparing the pool of primordial follicles in ovaries harvested from 5 year old fertile versus infertile cows, Erickson [2] reported over a 6-fold difference ( 119,000 versus 18,000, respectively). The number of growing follicles is an indirect measure of the pool of primordial follicles within the ovaries [3]. Ireland et al. [4] found a 7-fold difference in the number of antral follicles of young adult cattle but noted consistency within individuals for the number of antral follicles within follicular waves. If the pool of antral follicles available for stimulation explains the variation in superovulation, then quantification of antral follicles could be used to predict and possibly adjust superovulatory treatments for more consistent response.

Ultrasonography can be used to quantify the number of antral follicles present in the ovaries at the initiation of superovulation, but requires experienced technicians, expensive equipment and careful evaluation of ultrasound images. Anti-Mullerian hormone (AMH) is a glycoprotein that is expressed in granulosa cells of growing follicles [5] [6]. Secretion of AMH is greatest in 2 to $5 \mathrm{~mm}$ follicles [7], which represent the pool of follicles that would likely respond to superovulatory treatment. Anti-Mullerian hormone has been classified as a good predictive marker of the ovarian response to follicular stimulation for oocyte retrieval and in vitro embryo production [8] [9], as well as an endocrine marker that could help predict superovulatory responses of cows [10]. The present study was conducted to further investigate the use of AMH and/or follicle counts as a predictor of subsequent superovulatory response and embryo production in beef cows. 


\section{Materials and Methods}

\subsection{Animals, Ultrasonography and Blood Collection}

A total of 79 cows, including 31 Angus, 2 Chianina, 10 Polled Hereford, 1 Maine-Anjou, 15 Shorthorn, 3 Simmental, and 17 crossbred of various breeds were housed at the Food Animal Veterinary Services donor care facility located in Rensselaer, Indiana. Animal care was under the supervision of the veterinary staff at the donor care facility, following standard veterinary practice and animal welfare guidelines. The embryo donors consisted of 14 yearling to 2 year old heifers, 24 head of 3 to 5 year old cows, 27 head of 6 to 8 year old cows, and 14 head of 9 to 13 year old cows. Donor body condition score (BCS; 1 to 9 scale) [11] ranged from 4 to 8 , and averaged 6.0. Before the initiation of superovulation, ultrasonography (Aloka $500 \mathrm{~V}$ with $5.0 \mathrm{MHz}$ linear transducer; Corometrics, Wallingford, $\mathrm{CN}$ ) was used to scan the ovaries of each donor to record the number of 3 to $5 \mathrm{~mm}$ follicles present. Concurrent with ultrasonography, a $10 \mathrm{~mL}$ blood sample was collected via tail vein from each cow, using a BD Vacutainer SS Plus Blood Collection Tube (Ref. No. 367985, Becton, Dickson, Franklin Lakes, NJ). After clotting and centrifugation, recovered serum was placed in $5 \mathrm{~mL}$ polypropylene tubes and stored in a chest freezer at $-15^{\circ} \mathrm{C}$ to $-20^{\circ} \mathrm{C}$ until analysis for AMH.

\subsection{Superovulation Regimens and Insemination}

Depending upon scheduling, client preference and donor history, superovulatory treatment was initiated either during the luteal phase of cow's natural estrous cycle, or after insertion of a 1.38 g progesterone insert (CIDR; EAZI-Breed CIDR; Zoetis, Florham Park, NJ). Cows superovulated based on their natural cycle had superovulatory treatment initiated between Day 8 and 12 of the estrous cycle. Cows received twice daily (10 to 12 hours apart), decreasing doses of porcine follicle stimulating hormone (FSH; Follitropin-V, Bioniche Animal Health, Belleville Ontario CA) over a 4-day period. The cows were injected (i.m) with 50 mg (NIH-FSH-P1 units) of FSH twice daily the first 2 days, then 40 and $30 \mathrm{mg}$ twice daily on days 3 and 4, respectively. On the morning of the fourth day of FSH treatment, cows received an injection of $2.5 \mathrm{cc}(\sim 625 \mu \mathrm{g})$ of Cloprostenol (Estrumate, Merck Animal Health, Summit, NJ) to induce luteal regression. After Cloprostenol treatment, cows were observed until onset of estrus.

Cows superovulated regardless of the day of the estrous cycle, received an EAZI-Breed CIDR on Day 0, in conjunction with an injection (i.m.) of $2.5 \mathrm{mg}$ estradiol and $50 \mathrm{mg}$ of progesterone to suppress the dominant follicle and initiate a new follicular wave [12]. On Day 4, superovulatory treatment was initiated at follicular wave emergence, following the same 4-day, descending dose protocol used for cows during their natural cycle. On the morning of the fourth day of FSH treatment, the CIDR was removed at the time of Cloprostenol injection, and cows were observed until onset of estrus.

Timing of insemination after detected estrus depended on the number of 
straws and type of frozen-thawed semen used. When a single straw of semen was used, insemination occurred 12 to 16 hours after detected estrus. When multiple straws of semen were used, insemination occurred within 12 hours of detected estrus, and again 6 to 8 hours later. When X- or Y-sorted semen was used, insemination occurred 16 and again at 24 hours after detected estrus, with 3 to 6 straws of semen used in total, depending on sperm concentration per straw.

\subsection{Embryo Recovery and Evaluation}

Non-surgical embryo recoveries were performed $\sim$ Day 7 of the subsequent estrous cycle, using a Foley catheter and ViGRO complete flush medium (ViGRO ${ }^{\text {TM }}$, Bioniche Animal Health, Pullman, WA). At the time of embryo collection, the ovaries of donor cows were palpated to determine the number of corpora lutea (CL) present on each ovary. Embryos recovered from the flush medium were evaluated for stage of development (morula, early blastocyst or blastocyst) and morphological quality (grade 1, 2, degenerate or unfertilized), using evaluation criteria established by the International Embryo Transfer Society [13].

\subsection{Analysis of Serum Anti-Mullerian Hormone}

Serum samples were shipped on dry ice over night to the Texas A\&M Veterinary Medical Diagnostic Laboratory at College Station, TX. The diagnostic laboratory used a bovine AMH enzyme-linked immunosorbent assay kit (Bovine AMH ELISA AL-114; Ansh Labs, Webster, TX) to determine AMH concentration $(\mathrm{ng} / \mathrm{mL})$ in duplicate samples, following the assay kit manufacturer's recommended procedures. The AMH assay had an analytical sensitivity of 0.011 $\mathrm{ng} / \mathrm{mL}$

\subsection{Statistical Analysis}

Analysis was performed, using JMP Pro 12.0 statistical software (SAS Institute, Inc.). Variables considered in the analysis were embryo donor breed, superovulation protocol, $\mathrm{AMH}$ concentration, follicle and CL number, total, transferable (grade 1 or 2), degenerate embryos, and unfertilized ova. Superovulation protocol had no effect $(P \geq 0.293)$ on number of corpora lutea, or the number of total, transferrable, degenerate embryos or unfertilized ova, so was removed from the analysis. There were no breed differences $(\mathrm{P}=0.321)$ for serum AMH concentration.

Frequency distribution was used to assign AMH concentration measured in serum samples to quartiles. Analysis of variance was then used to make comparisons between $\mathrm{AMH}$ quartiles for number of 3 to $5 \mathrm{~mm}$ follicles, number of corpora lutea at embryo collection, number of embryos recovered, and the percentages of transferrable and degenerate embryos, and unfertilized ova. Percent transferrable embryos were defined as the portion of total embryos recovered that were of grade 1 or 2 morphological quality. Percent degenerate embryos 
were defined as the portion of total embryos recovered that exhibited limited cleavage and/or were of poor morphological quality. Percent unfertilized ova were the portion of all recovered embryos/structures that exhibited no cleavage.

Frequency distribution was also used to assign follicle counts to quartiles. Analysis of variance was then used to make comparisons between follicle quartiles and number of corpora lutea at embryo collection, number of embryos recovered, and the percentages of transferrable and degenerate embryos, and unfertilized ova. Multivariate analysis was used to determine any correlations between AMH concentrations and number of 3 to $5 \mathrm{~mm}$ follicles, number of corpora lutea at embryo collection, number of embryos recovered, and the percentages of transferrable and degenerate embryos, and unfertilized ova. All values are expressed as the mean \pm SEM. Statistical differences were considered significant where $\mathrm{P}<0.05$.

\section{Results}

\subsection{Anti-Mullerian Hormone as a Predictor of Superovulatory Response}

Anti-Mullerian hormone measured in serum samples ranged from 0.013 to 0.898 $\mathrm{ng} / \mathrm{mL}$, with a mean of $0.293 \mathrm{ng} / \mathrm{mL}$. The distribution of AMH concentrations was divided into quartiles (AMH Q1 through Q4, with Q1 the lowest and Q4 the highest $\mathrm{ng} / \mathrm{mL}$ ) for analysis. The range of AMH concentrations within each quartile are presented in Table 1 . The assay failed to detect AMH in 2 serum samples. Therefore, 77 or 79 embryo donors were included in Table 1, with embryos collected from 20 donors twice ( 97 total embryo collections). Donor cows in AMH Q4 had a greater $(\mathrm{P}<0.001)$ number of 3 to $5 \mathrm{~mm}$ follicles at the start of superovulation than did donors in either Q1 or Q2. Cows in AMH Q3 were intermediate for mean number of follicles. At the time of embryo collection, cows in AMH Q3 and 4 had more palpable CL than cows in AMH Q1 (P < 0.001). The mean number of CL for cows in AMH Q2 was intermediate and similar $(\mathrm{P}>0.10)$ to those in both $\mathrm{AMH} \mathrm{Q} 1$ and 3. The mean number of embryos recovered for donor cows in AMH Q4 was greater $(\mathrm{P}<0.001)$ than those recovered from cows in either AMH Q1 or 2, but similar to that of AMH Q3. The percentage of recovered embryos that were classified as transferrable, degenerate or unfertilized were similar $(\mathrm{P} \geq 0.275)$ across AMH quartiles. Multivariate analysis (Table 2$)$ indicated that $\mathrm{AMH}$ was positively correlated $(\mathrm{P}<0.001)$ with mean follicles $(r=0.458), C L(r=0.452)$ and embryos recovered $(r=$ $0.430)$.

\subsection{Antral Follicle Number as a Predictor of Superovulatory Response}

The number of 3 to $5 \mathrm{~mm}$ follicles counted on the ovaries of donor cows ranged from 5 to over 30, with a mean of 16 . In order to determine if follicle counts at the start of superovulation might be predictive of subsequent superovulatory 
Table 1. Quartile categorization of AMH concentrations as a predictor of superovulatory outcomes in beef cattle.

\begin{tabular}{|c|c|c|c|c|c|}
\hline \multirow{3}{*}{$\begin{array}{c}\text { Item } \\
\mathrm{AMH}, \mathrm{ng} / \mathrm{mL}\end{array}$} & \multicolumn{4}{|c|}{ Quartile of AMH concentration } & \multirow{3}{*}{ P-value } \\
\hline & Q1 & Q2 & Q3 & Q4 & \\
\hline & $0.013-0.168$ & $0.169-0.263$ & $0.264-0.363$ & $0.364-0.898$ & \\
\hline No. of donors/collections ${ }^{*}$ & 26 & 23 & 24 & 24 & \\
\hline No. of follicles & $13.46 \pm 0.91^{\mathrm{b}}$ & $14.95 \pm 0.98^{\mathrm{b}}$ & $16.79 \pm 0.94^{\mathrm{ab}}$ & $19.33 \pm 0.94^{\mathrm{a}}$ & 0.001 \\
\hline No. of CL & $11.62 \pm 1.54^{\mathrm{c}}$ & $13.68 \pm 1.67^{\mathrm{bc}}$ & $17.58 \pm 1.60^{\mathrm{ab}}$ & $20.54 \pm 1.60^{\mathrm{a}}$ & 0.001 \\
\hline No. of embryos & $9.77 \pm 1.76^{\mathrm{b}}$ & $9.36 \pm 1.91^{\mathrm{b}}$ & $15.50 \pm 1.83^{\mathrm{ab}}$ & $20.13 \pm 1.83^{\mathrm{a}}$ & 0.001 \\
\hline Transferable \% & $69.32 \pm 6.62$ & $57.06 \pm 7.08$ & $58.75 \pm 6.62$ & $51.05 \pm 6.62$ & 0.275 \\
\hline Degenerate \% & $5.52 \pm 2.52$ & $7.89 \pm 2.69$ & $9.40 \pm 2.52$ & $9.87 \pm 2.52$ & 0.614 \\
\hline Unfertilized \% & $25.16 \pm 6.73$ & $35.06 \pm 7.19$ & $31.85 \pm 6.73$ & $39.09 \pm 6.73$ & 0.519 \\
\hline
\end{tabular}

*Included 77 of 79 donors, with 20 donors collected twice; ${ }^{\mathrm{a}, \mathrm{b}, \mathrm{c}}$ Means \pm S.E.M within rows with different superscripts are significantly different $(\mathrm{P}<0.05)$.

Table 2. Correlations between AMH, follicle counts, and embryo production of superovulated donor cows.

\begin{tabular}{|c|c|c|c|}
\hline Variable 1 & Variable 2 & Correlation & $\mathrm{P}$-value \\
\hline \multirow[t]{6}{*}{ Anti-Mullerian hormone } & Mean number of follicles & 0.458 & 0.001 \\
\hline & Mean number of corpora lutea & 0.452 & 0.001 \\
\hline & Mean number of embryos & 0.430 & 0.001 \\
\hline & Percent transferrable embryos & -0.126 & 0.231 \\
\hline & Percent degenerate embryos & 0.195 & 0.061 \\
\hline & Percent unfertilized ova & 0.052 & 0.621 \\
\hline \multirow[t]{5}{*}{ Mean number of follicles } & Mean number of corpora lutea & 0.556 & 0.001 \\
\hline & Mean number of embryos & 0.423 & 0.001 \\
\hline & Percent transferrable embryos & -0.037 & 0.724 \\
\hline & Percent degenerate embryos & 0.147 & 0.153 \\
\hline & Percent unfertilized ova & -0.018 & 0.857 \\
\hline \multirow[t]{4}{*}{ Mean number of corpora lutea } & Mean number of embryos & 0.887 & 0.001 \\
\hline & Percent transferrable embryos & -0.008 & 0.942 \\
\hline & Percent degenerate embryos & 0.191 & 0.063 \\
\hline & Percent unfertilized ova & -0.064 & 0.539 \\
\hline \multirow[t]{3}{*}{ Mean number of embryos } & Percent transferrable embryos & 0.011 & 0.919 \\
\hline & Percent degenerate & 0.236 & 0.021 \\
\hline & Percent unfertilized & -0.098 & 0.341 \\
\hline \multirow[t]{2}{*}{ Percent transferrable embryos } & Percent degenerate embryos & -0.184 & 0.073 \\
\hline & Percent unfertilized ova & -0.931 & 0.001 \\
\hline Percent unfertilized ova & Percent degenerate & -0.188 & 0.067 \\
\hline
\end{tabular}


response, the distribution of follicle counts were also divided into quartiles (F Q1 through Q4, with Q1 the lowest and Q4 the highest) for analysis (Table 3). Donor cows with higher follicle counts (F Q3 and 4) at the start of superovulation had more $(\mathrm{P}<0.001)$ palpable $\mathrm{CL}$ at embryo collection than donor cows in $\mathrm{F}$ Q1 or 2. More embryos were recovered from cows with the highest follicle counts (F $\mathrm{Q} 4)$ as compared with cows having lower (F Q1 and 2) follicle counts ( $<$ 0.001). The number of embryos recovered from donors in $F$ Q3 was intermediate and similar $(\mathrm{P}>0.10)$ to that of donors in F Q1, 2 and 4. The percentage of transferable embryos and unfertilized ova were similar $(\mathrm{P} \geq 0.688)$ across follicle count quartiles. The mean percentage of degenerate embryos was greater for donor cows in F Q3 than any other follicle quartile $(\mathrm{P}=0.002)$.

As was noted for $\mathrm{AMH}$, mean follicles at the start of superovulation was positively correlated $(\mathrm{P}<0.001$; Table 2$)$ with mean $\mathrm{CL}(\mathrm{r}=0.556)$ and mean embryos $(r=0.423)$ but not percentage of viable or degenerate embryos, or unfertilized oocytes $(\mathrm{P} \geq 0.153)$. Other correlations noted were positive correlations between mean CL and embryos recovered $(r=0.887$; $\mathrm{P}<0.001)$, and between embryos collected and degenerate embryos $(r=0.236 ; \mathrm{P}=0.021)$. As might be expected, a significant negative correlation $(r=-0.931$; $P<0.001)$ existed between percentage viable and percentage unfertilized embryos.

\section{Discussion}

The objective of the present study was to further investigate the use of AMH and/or follicle counts as a predictor of subsequent superovulatory response and embryo production in beef cows. The goal of superovulation is to stimulate a number of small antral follicles to grow and mature, resulting in multiple ovulations. Therefore, the pool of small antral follicles available for stimulation should dictate the superovulatory response. Ireland et al. [4] used daily ultrasound to count the number of $3 \mathrm{~mm}$ or larger follicles present in the ovaries of 90 beef heifers over 2 consecutive follicular waves. Results indicated that follicle numbers

Table 3. Quartile categorization of follicle counts as a predictor of superovulatory response and embryo production.

\begin{tabular}{|c|c|c|c|c|c|}
\hline \multirow{2}{*}{ Item } & \multicolumn{4}{|c|}{ Quartile of follicle counts } & \multirow{2}{*}{ P-value } \\
\hline & Q1 & Q2 & Q3 & Q4 & \\
\hline No. of donors & 26 & 35 & 20 & 18 & \\
\hline Follicle range & $5-12$ & $13-17$ & $18-20$ & $21-30$ & \\
\hline No. of CL & $10.65 \pm 1.40^{\mathrm{b}}$ & $13.51 \pm 1.20^{\mathrm{b}}$ & $19.15 \pm 1.59^{\mathrm{a}}$ & $23.33 \pm 1.68^{\mathrm{a}}$ & 0.001 \\
\hline No. of embryos & $9.62 \pm 1.79^{b}$ & $11.57 \pm 1.55^{\mathrm{b}}$ & $16.50 \pm 2.04^{\mathrm{ab}}$ & $20.00 \pm 2.16^{\mathrm{a}}$ & 0.001 \\
\hline Transferable \% & $58.20 \pm 6.44$ & $64.77 \pm 5.63$ & $54.51 \pm 7.53$ & $58.46 \pm 7.97$ & 0.716 \\
\hline Degenerate \% & $4.82 \pm 2.23^{\mathrm{b}}$ & $6.71 \pm 1.95^{\mathrm{b}}$ & $17.09 \pm 2.61^{\mathrm{a}}$ & $5.38 \pm 2.76^{\mathrm{b}}$ & 0.002 \\
\hline Unfertilized \% & $36.99 \pm 6.44$ & $28.53 \pm 5.63$ & $28.39 \pm 7.54$ & $36.16 \pm 7.97$ & 0.688 \\
\hline
\end{tabular}

${ }^{\mathrm{a}, \mathrm{b}}$ Means \pm S.E.M within rows with different superscripts are significantly different $(\mathrm{P}<0.05)$. 
were highly variable between individual animals, but highly repeatable $(r=0.89)$ within individual heifers for average number of follicles per follicular wave. Likewise, AMH in circulation, which is a reflection of the number of growing follicles within the ovaries, is repeatable within individuals. Ireland et al. [14] measured AMH daily in young adult beef heifers from 4 days before to 4 days after ovulation. The day-to-day variation in AMH concentration was relatively minor within individuals. Results indicated that a single measure of $\mathrm{AMH}$, regardless of the day of the estrous cycle when measured, accurately reflected serum AMH concentration. Subsequent studies [10] [15] [16] in dairy cows confirmed that within individuals, AMH concentration is relatively constant over time.

Assay of AMH in the serum of 77 embryo donor beef cows in the present study found a range of 0.013 to $0.898 \mathrm{ng} / \mathrm{mL}$. This range of serum AMH is somewhat broader than that reported by others. Ireland et al. [14] reported an AMH range of $\sim 0.05$ to $0.50 \mathrm{ng} / \mathrm{mL}$ for beef heifers. Rico et al. [15] reported that AMH ranged from 0.049 to $0.359 \mathrm{ng} / \mathrm{mL}$ at estrus and 0.026 to $0.212 \mathrm{ng} / \mathrm{ml} 7$ days after estrus in dairy cows. Monniaux et al. [17] reported AMH concentrations to range from 0.001 to $0.531 \mathrm{ng} / \mathrm{mL}$, while Souza et al. [16] reported an AMH range from 0 to $0.374 \mathrm{ng} / \mathrm{mL}$ in dairy cows. A difficulty is using $\mathrm{AMH}$ as a predictor of superovulatory response is in how to classify a donor cow based on an AMH assay. Different AMH assays, the use of EDTA versus heparinized plasma versus serum, and storage conditions can alter results [10]. Rico et al. [10] reported AMH concentrations in Holstein cows ranging from 0.005 to 0.244 $\mathrm{ng} / \mathrm{mL}$ where cattle below $0.087 \mathrm{ng} / \mathrm{mL}$ were identified as poor responders to superovulation, as defined as having less than 15 large follicles at estrus. Using similar criteria for poor responders to superovulation, Souza et al. [16] suggested a cut-off value of $0.130 \mathrm{ng} / \mathrm{mL} \mathrm{AMH}$.

Different AMH assays, blood sample types, sample storage conditions, and criteria for measuring superovulatory response can alter recommended AMH cut-off values for selecting potential donor cows. Whereas other studies utilized animals of similar age and breeding, embryo donors in the present study ranged from breeding age heifers to 13-year-old cows, and were of various beef breeds. Therefore, embryo donors were classified into quartiles of circulating $\mathrm{AMH}$ concentration rather than by specific concentrations in blood. Embryo donor cows in the lowest AMH quartile $(0.013$ to $0.168 \mathrm{ng} / \mathrm{mL}$ ) had fewer follicles at the start of superovulation, fewer palpable CL at embryo collection, and produced fewer embryos than donors in the highest AMH quartile. Donors in AMH quartiles 2 and 3 were intermediate for follicle, CL and embryo counts. Souza et al. [16] assigned dairy donor cows to AMH quartiles and reported cows in the highest AMH quartile produced more total embryos or ova, more transferrable and freezable embryos than cows in the lowest quartile. Similarly, Monniaux et al. [17] reported that superovulated dairy cows with AMH $>0.20 \mathrm{ng} / \mathrm{mL}$ or 0.10 to $0.20 \mathrm{ng} / \mathrm{mL}$ produced more total and transferrable embryos than cows in the 
lowest $(<0.10 \mathrm{ng} / \mathrm{mL})$ AMH category. There were not differences $(\mathrm{P}=0.275)$ among AMH quartiles in the current study for percentage of embryos collected that were of transferrable quality. Numerically, donor cows in the highest AMH quartile produced more transferrable embryos, but not on a percentage basis.

In this study, AMH in circulation was positively correlated $(\mathrm{P}=0.001)$ with follicles present at start of superovulation $(\mathrm{r}=0.458)$, and number of CL $(\mathrm{r}=$ $0.452)$ and embryos recovered at collection $(r=0.430)$. Rico et al. [15] reported positive correlations of 0.79 and 0.64 between $\mathrm{AMH}$ in circulation and the number of 3 to $7 \mathrm{~mm}$ follicles before and ovulations after superovulation, respectively. In a later study, Rico et al. [10] reported positive correlations between AMH in plasma and large follicles at estrus $(r=0.46)$ and CL number $(r=0.43)$ in superovulated cows. Souza et al. [16] reported similar positive correlations between circulating AMH and CL number $(r=0.65)$ and embryos/ova recovered $(\mathrm{r}=0.50)$ after superovulation, but a lower correlation to number of transferrable embryos $(\mathrm{r}=0.28)$. Monniaux et al. [17] also reported positive correlations of 0.49 and 0.32 between plasma AMH and the number of total and transferrable embryos recovered, respectively. Overall, these results are in agreement that circulating AMH has significant positive correlations with follicle number, ovulation rate and number of embryos recovered.

A difference between the present study and others [16] [17] was a lack of correlation between $\mathrm{AMH}$ and embryo morphological quality, as reflected by the percentage of transferrable embryos. However, results are in agreement with Ireland et al. [4] who reported that heifers with high follicle numbers $(\geq 25)$ produced more transferrable embryos, but not a higher proportion of transferrable embryos. The results of Souza et al. [16] are in agreement that there is no significant correlation between circulating AMH and fertilization rate. Fertilization rate can vary, depending on semen quality and concentration, insemination timing and technique, and inherit fertility of the bull. Embryo morphological quality after fertilization can be influenced by the same factors, as well as uterine environment. Superovulation can alter follicular development, oocyte maturation, ovulation and sperm transport. These abnormalities may disrupt the normal fertilization and/or embryo development to result in an increased number of unfertilized oocytes and poor quality embryos [18].

Assay for AMH is relatively expensive and limited in availability to most embryo transfer practitioners. However, many practitioners have access to ultrasonography and often evaluate ovarian structures before superovulation. As an alternate to $\mathrm{AMH}$, the number of 3 to $5 \mathrm{~mm}$ follicles present in the ovaries at initiation of superovulatory treatment was evaluated as a predictor of superovulatory response. As with serum $\mathrm{AMH}$, donors were assigned to quartiles for comparison. Results indicate that follicle counts are of value for predicting subsequent superovulatory response. Donor cows in the highest two quartiles for 3 to $5 \mathrm{~mm}$ follicles had more ovulations, based on CL present at embryo collection than cows in the lowest two follicle quartiles. Donors in the highest follicle quartile 
also produced more embryos that cows in the lowest two quartiles. As with circulating $\mathrm{AMH}$, mean number of follicles at start of superovulation was positively correlated $(\mathrm{P}=0.001)$ with ovulations $(\mathrm{CL})$ after superovulation $(\mathrm{r}=0.556)$ and number of embryos recovered $(\mathrm{r}=0.423)$. In a previous study using ultrasound to determine antral follicle count, Ireland et al. [4] reported that cows with high follicle numbers also had more embryos recovered and more transferable embryos.

Currently, embryo donor information such as breed, age, parity and past superovulatory response are used to adjust the superovulation regimen in an effort to improve embryo production. While improvements have been made in hormonal treatment and synchronization protocols [1] the mean number of transferrable embryos recovered per donor animal has remained relatively constant for the past 40 years. Many commercial embryo transfer (ET) programs are limited in options because donors and service sires are often chosen by the owner, and many times procedures are done on farm and mostly out of the control of practitioners. The ability to make adjustments to superovulatory regimens based on predicted superovulatory response of individual donors would be of great benefit to the ET industry moving forward. This study confirms that relative $\mathrm{AMH}$ level is positively correlated with number of small antral follicles in the ovaries of cows and might be used to either predict superovulatory response or possibly adjust superovulatory regimen to improve superovulatory response. Antral follicle counts at the initiation of superovulatory treatments might be a more practical alternate to AMH for embryo transfer practitioners to use in predicting superovulatory response. Further study is needed to determine the effectiveness of using either AMH assay or follicle counts to adjust superovulatory regimens for improved response. For example, it might be advisable to extend the time of gonadotropin treatment for individuals with lower follicle counts in order to improve response. Likewise, the gonadotropin dose or duration of treatment might be reduced in individuals with a large number of follicles to avoid overstimulation, which often results in poor embryo quality.

\section{Acknowledgements}

The authors acknowledge Dr. Scott Jacques at the Texas A\&M Veterinary Diagnostic Laboratory for performing Anti-Mullerian hormone assays, and the University of Arkansas Agricultural Experiment Station and the Department of Animal Science for their financial support.

\section{References}

[1] Hasler, J.F. (2014) Forty Years of Embryo Transfer in Cattle: A Review Focusing on the Journal Theriogenology, the Growth of the Industry in North America, a Personal Reminisces. Theriogenology, 81, 152-169. https://doi.org/10.1016/j.theriogenology.2013.09.010

[2] Erickson, B.H. (1966) Development and Senescence of the Postnatal Bovine Ovary. Journal of Animal Science, 25, 800-805. https://doi.org/10.2527/jas1966.253800x 
[3] Scheffer G.J., Broekmans, F.J., Dorland, M., Habbema, J.D., Looman, C.W. and te Velde, E.R. (1999) Antral Follicle Counts by Transvaginal Ultrasonography Are Related to Age in Women with Proven Natural Fertility. Fertility and Sterility, 72, 845-851. https://doi.org/10.1016/S0015-0282(99)00396-9

[4] Ireland, J.J., Ward, F., Jimenez-Krassel, F., Ireland, J.L.H., Smith, G.W., Lonergan, P. and Evans, A.C.O. (2007) Follicle Numbers Are Highly Repeatable within Individual Animals but Are Inversely Correlated with FSH Concentrations and the Proportion of Good-Quality Embryos after Ovarian Stimulation in Cattle. Human Reproduction, 22, 1687-1695. https://doi.org/10.1093/humrep/dem071

[5] Vigier, B., Picard, J.Y., Tran, D., Legeai, L. and Josso, N. (1984) Production of Anti-Müllerian Hormone: Another Homology between Sertoli and Granulosa Cells. Endocrinology, 114, 1315-1320. https://doi.org/10.1210/endo-114-4-1315

[6] Takahashi, M., Hayashi, M., Manganaro, T.F. and Donahoe, P.K. (1986) The Ontogeny of Mullerian Inhibiting Substance in Granulosa Cells of the Bovine Ovarian Follicle. Biology of Reproduction, 35, 447-453. https://doi.org/10.1095/biolreprod35.2.447

[7] Van Rooij, I.A., Broekmans, F.J., Te Velde, E.R., Fauser, B.C., Bancsi, L.F., de Jong, F.H. and Themmen, A.P. (2002) Serum Anti-Müllerian Hormone Levels: A Novel Measure of Ovarian Reserve. Human Reproduction, 17, 3065-3071.

https://doi.org/10.1093/humrep/17.12.3065

[8] Muttukrishna, S., Suharjono, H., McGarrigle, H. and Sathanandan, M. (2004) Inhibin B and Anti-Mullerian Hormone: Markers of Ovarian Response in IVF/ICSI Patients? BJOG: An International Journal of Obstetrics \& Gynaecology, 111, 1248-1253. https://doi.org/10.1111/j.1471-0528.2004.00452.x

[9] Muttukrishna, S., McGarrigle, H., Wakim, R., Khadum, I., Ranieri, D.M. and Serhal, P. (2005) Antral Follicle Count, Anti-Mullerian Hormone and Inhibin B: Predictors of Ovarian Response in Assisted Reproductive Technology? BJOG: An International Journal of Obstetrics \& Gynaecology, 112, 1384-1390. https://doi.org/10.1111/j.1471-0528.2005.00670.x

[10] Rico, C., Drouilhet, L., Salvetti, P., Dalbiès-Tran, R., Jarrier, P., Touzé, J.L., Pillet, E., Ponsart, C., Fabre, S. and Monniaux, D. (2012) Determination of Anti-Müllerian Hormone Concentrations in Blood as a Tool to Select Holstein Donor Cows for Embryo Production: From the Laboratory to the Farm. Reproduction, Fertility and Development, 24, 932-944. https://doi.org/10.1071/RD11290

[11] Wagner, J.J., Lusby, K.S., Oltjen, J.W., Rakestraw, J., Wettemann, R.P. and Walters, L.E. (1988) Carcass Composition in Mature Hereford Cows: Estimation and Effect on Daily Metabolizable Energy Requirement during Winter. Journal of Animal Science, 66, 603-612. https://doi.org/10.2527/jas1988.663603x

[12] Bo, G.A., Adams, G.P., Pierson, R.A. and Mapletoft, R.J. (1995) Exogenous Control of Follicular Wave Emergence in Cattle. Theriogenology, 43, 31-40. https://doi.org/10.1016/0093-691X(94)00010-R

[13] Stringfellow, D.A. and Seidel, S.M. (1998) Manual of the International Embryo Transfer Society: A Procedural Guide and General Information for the Use of Embryo Transfer Technology Emphasizing Sanitary Procedures. 3rd Edition, International Embryo Transfer Society, Savory, IL.

[14] Ireland, J.L., Scheetz, D., Jimenez-Krassel, F., Themmen, A.P., Ward, F., Lonergan, P., Smith, G.W., Perez, G.I., Evans, A.C. and Ireland, J.J. (2008) Antral Follicle Count Reliably Predicts Number of Morphologically Healthy Oocytes and Follicles in Ovaries of Young Adult Cattle. Biology of Reproduction, 79, 1219-1225.

https://doi.org/10.1095/biolreprod.108.071670 
[15] Rico, C., Fabre, S., Médigue, C., di Clemente, N., Clément, F., Bontoux, M., Touzé, J.L., Dupont, M., Briant, E., Rémy, B., Beckers, J.F. and Monniaux, D. (2009) Anti-Müllerian Hormone Is an Endocrine Marker of Ovarian Gonadotropin-Responsive Follicles and Can Help to Predict Superovulatory Responses in the Cow. Biology of Reproduction, 80, 50-59. https://doi.org/10.1095/biolreprod.108.072157

[16] Souza, A.H., Carvalho, P.D., Rozner, A.E., Vieira, L.M., Hackbart, K.S., Bender, R.W., Dresch, A.R., Verstegen, J.P., Shaver, R.D. and Wiltbank, M.C. (2015) Relationship between Circulating Anti-Mullerian Hormone (AMH) and Superovulatory Response of High-Producing Dairy Cows. Journal of Dairy Science, 98, 169-178. https://doi.org/10.3168/jds.2014-8182

[17] Monniaux, D., Barbey, S., Rico, C., Fabre, S., Gallard, Y. and Larroque, H. (2010) Anti-Müllerian Hormone: A Predictive Marker of Embryo Production in Cattle? Reproduction, Fertility and Development, 22, 1083-1091. https://doi.org/10.1071/RD09279

[18] Kafi, M. and McGowan, M.R. (1997) Factors Associated with Variation in the Superovulatory Response of Cattle. Animal Reproduction Science, 48, 137-157. https://doi.org/10.1016/S0378-4320(97)00033-X 